\title{
High similarity of Trypanosoma cruzi kDNA genetic profiles detected by LSSP-PCR within family groups in an endemic area of Chagas disease in Brazil
}

\author{
Sandra Maria Alkmim-Oliveira ${ }^{[1]}$, Henrique Borges Kappel ${ }^{[1]}$, Cristiane Pontes Andrade ${ }^{[1]}$, \\ Aluízio Prata ${ }^{[2]}$, Luis Eduardo Ramirez ${ }^{[1]}$, Dalmo Correia $^{[2]}$ and Eliane Lages-Silva ${ }^{[1]}$
}

[1]. Disciplina de Parasitologia, Departamento de Ciências Biológicas, Universidade Federal do Triângulo Mineiro, Uberaba, MG. [2]. Disciplina de Doenças Infecciosas e Parasitárias, Departamento de Clínica Médica, Universidade Federal do Triângulo Mineiro, Uberaba, MG. † in memoriam.

\begin{abstract}
Introduction: Determining the genetic similarities among Trypanosoma cruzi populations isolated from different hosts and vectors is very important to clarify the epidemiology of Chagas disease. Methods: An epidemiological study was conducted in a Brazilian endemic area for Chagas disease, including 76 chronic chagasic individuals $(96.1 \%$ with an indeterminate form; $46.1 \%$ with positive hemoculture). Results: T. cruzi I (TcI) was isolated from one child and TcII was found in the remaining (97.1\%) subjects. Low-stringency single-specific-primer-polymerase chain reaction (LSSP-PCR) showed high heterogeneity among TcII populations (46\% of shared bands); however, high similarities (80-100\%) among pairs of mothers/children, siblings, or cousins were detected. Conclusions: LSSP-PCR showed potential for identifying similar parasite populations among individuals with close kinship in epidemiological studies of Chagas disease.
\end{abstract}

Keywords: Trypanosoma cruzi. Endemic area. LSSP-PCR.

Trypanosoma cruzi has a multiclonal structure with wide biological diversity and high genetic polymorphism. This heterogeneity is associated with the presence of different parasite populations in the same host, a broad geographic distribution, infection stage, and susceptibility or resistance to specific treatments ${ }^{1}$.

Trypanosoma cruzi populations can be divided into six distinct discrete typing units (DTUs) named $\mathrm{TcI}-\mathrm{VI}^{2}$, which show highly heterogeneity within and between types. TcII, TcV, and TcVI are associated with the domestic transmission cycle and are the main causes of Chagas disease in the southern and central regions of South America. TcI isolates are associated with wild and domestic cycles, and are frequently detected in the northern parts of the Amazon region and in endemic areas of Venezuela, Colombia, and Mexico. TcIV and TcIII circulate in the wild and are relatively poorly studied ${ }^{3}$.

The prevalence and geographic distributions of clinical forms of Chagas disease show differences among and within countries. These differences appear to be related to the genetic characteristics of hosts and strains of the parasite within a specific region. However, the data reported to date are

\footnotetext{
Address to: Prof. Luis Eduardo Ramirez Giraldo. Disc. de Parasitologia/ Dept $^{\circ}$ de Ciências Biológicas/UFTM. Av. Getúlio Guaritá s/n, 38025-440 Uberaba, MG, Brasil.

Phone: 5534 3318-5258; Fax: 5534 3318-5279.

e-mail: ramirez_fmtm@yahoo.com.br; salkmim@hotmail.com

Received 4 December 2013

Accepted 26 May 2014
}

inconclusive, making assessment of epidemiological studies difficult ${ }^{3}$.

Currently, the application of molecular techniques for epidemiological studies on Chagas disease has been proposed, which has stimulated research aiming to evaluate the relationship between the transmission mechanisms, DTUs of T. cruzi, and clinical forms of the disease ${ }^{3}$.

The low-stringency single-specific-primer-polymerase chain reaction (LSSP-PCR) technique has been successfully applied for T. cruzi characterization. This method has shown excellent potential for evaluating intraspecific differences or similarities of $T$. cruzi populations and shows stability and reproducibility in experimental and human studies ${ }^{4}$.

In this study, the epidemiological application of LSSP-PCR in the characterization of TcII samples demonstrated similar genetic profiles circulating among individuals with very close kinship. Seventy-six chronic Chagas disease individuals from an endemic post-control vector program area in the State of Bahia (Brazil) were evaluated; the age of subjects ranged from 2 to 56 years (mean, $31.62 \pm 12.25$ years). Trypanosoma cruzi infection was detected by positive serology of anti-T. cruzi using indirect immunofluorescence and an enzyme-linked immunosorbent assay. This research project was approved by the Ethics Committee ( ${ }^{\circ} 388$ ) of the Universidade Federal do Triângulo Mineiro (UFTM) and all procedures were carried out with the informed consent of patients.

The clinical records of the patients were classified according to their symptoms and electrocardiographic and radiological abnormalities (esophagogram, opaque enema, and chest X-ray); 
$96.1 \%$ of patients presented the indeterminate form and $4 \%$ presented the cardiac form of Chagas disease.

Parasitemia and parasite isolation were evaluated by hemoculture 5 and demonstrated the presence of $T$. cruzi in $46.1 \%$ $(35 / 76)$ of the individuals, without significant differences with respect to gender ( $40 \%$ male and $60 \%$ female) and age group (range, $4-56$ years; mean, $31.43 \pm 11.95$ years) ( $p>0.05)$. The degree of kinship in the study population was also determined, and 13 pairs of the 35 individuals with positive hemoculture showed kinship.

Deoxyribonucleic acid (DNA) extraction of the positive hemoculture samples preserved in guanidineethylenediaminetetraacetic acid was performed in duplicate by the phenol-chloroform method ${ }^{6}$. Trypanosoma cruzi DNA controls represented by negative and positive samples were included in all DNA extractions and PCR procedures.

Genetic characterization of $T$. cruzi kinetoplast-DNA (kDNA) was performed by LSSP-PCR in two steps ${ }^{4}$. The first step consisted of specific amplification of a variable region of $T$. cruzi minicircles, using the primers 121 (5'-AAATAATGTACGGGGGAGATGCATGA-3') and 122 (5'-GGTTCGATTGGGGTTGGTGTAATATA- $\left.3^{\prime}\right)^{7}$. The PCR was carried out in a final volume of $20 \mu \mathrm{L}$ containing $10 \mathrm{mM}$ Tris- $\mathrm{HCl}$ (pH 9.0), $0.1 \%$ Triton X-100, $3.5 \mathrm{mM} \mathrm{MgCl}_{2}$, $75 \mathrm{mM} \mathrm{KCl}, 0.2 \mathrm{mM}$ each of deoxyadenosine triphosphate (dATP), deoxycytidine triphosphate (dCTP), deoxyguanosine triphosphate (dGTP), and deoxythymidine triphosphate (dTTP), 1.0 unit of Taq DNA polymerase (Promega; Madison, WI, USA), $20 \mathrm{pmol}$ of each primer, $2 \mu \mathrm{L}(1 \mathrm{ng} / \mu \mathrm{L})$ of DNA, and $30 \mu \mathrm{L}$ of mineral oil. The amplification consisted of initial denaturing at $95^{\circ} \mathrm{C}(5 \mathrm{~min})$ and 35 cycles: $95^{\circ} \mathrm{C}(1 \mathrm{~min}), 65^{\circ} \mathrm{C}(1 \mathrm{~min})$, and $72^{\circ} \mathrm{C}(1 \mathrm{~min})$, followed by final extension at $72^{\circ} \mathrm{C}(10 \mathrm{~min})$. The PCR-amplified products were submitted to electrophoresis on a $1.5 \%$ agarose gel ( $1 \%$ agarose, $0.5 \%$ low-melting-point agarose) and stained with ethidium bromide. The 330-bp fragments of individual amplifications, corresponding to approximately 150ng of DNA, were excised from the gel, melted, diluted 10 -fold in double-distilled water, and used as the template for the second step of amplification. The second PCR was performed using a single S35 primer (5'-AAATAATGTACG GGGGAGATGCATGA-3') in a final volume of $10 \mu \mathrm{L}$, containing $10 \mathrm{mM}$ Tris- $\mathrm{HCl}(\mathrm{pH} 8.5), 0.1 \%$ Triton X-100, $1.5 \mathrm{mM} \mathrm{MgCl}{ }_{2}, 0.2 \mathrm{mM}$ each of dATP, dCTP, dGTP, and dTTP, 1.0 unit of Taq DNA polymerase (Promega; Madison, WI, USA), 45 pmol of the S35 primer, $3 \mu \mathrm{L}(1.5 \mathrm{ng} / \mu \mathrm{L})$ of DNA template, and $30 \mu \mathrm{L}$ of mineral oil. The amplification consisted of 40 cycles: $94^{\circ} \mathrm{C}(1 \mathrm{~min}), 30^{\circ} \mathrm{C}(1 \mathrm{~min})$, and $72^{\circ} \mathrm{C}(1 \mathrm{~min})$, preceded by initial denaturing at $94^{\circ} \mathrm{C}(5 \mathrm{~min})$ and followed by final extension at $72^{\circ} \mathrm{C}(7 \mathrm{~min})$. In order to demonstrate the stability of the amplification, each DNA sample was analyzed in duplicate. The LSSP-PCR products were separated by electrophoresis on a $7.5 \%$ polyacrylamide gel and stained with $0.2 \%$ silver nitrate. The genetic profiles obtained by visual scan photography of the gel were compared using the Nei and Li coefficient in GelCompar II versão 5.0 software (Fingerprint and Gel Analysis Software-Applied Maths NV).
Parasite DTUs were identified by amplification of the intergenic region of spliced leader genes, by amplification of the D7 domain of the 24S $\alpha$ ribosomal ribonucleic acid genes using nested-hot-start PCR assays and by nested amplification of the A-10 fragment, as previously reported ${ }^{8}$. Trypanosoma rangeli detection was performed using a multiplex PCR with the primers D72, D75, and RG3 ${ }^{9}$. For identification of T. cruzi DTUs, the PCR amplification products were viewed on a $6 \%$ polyacrylamide gel and the products of multiplex PCR were viewed on a $7.5 \%$ polyacrylamide gel stained with silver nitrate.

For the statistical analysis, the chi-squared test was used to determine the association between positive hemoculture with the patients' age group and gender. The Shapiro-Wilk test was used to investigate whether the age distributions of the groups was normal. If so, the Student's $t$-test for independent samples was performed. Associations between patient age groups and the complexity level of the genetic profiles for $T$. cruzi obtained from LSSP-PCR were investigated using regression analysis. The significance level used was $5 \%(p<0.05)$. The analyses were performed using the Statistica for Windows program, version 8.0 (StatSoft, Inc.; USA).

TcII populations were predominant and were detected in $97.1 \%$ (34/35) of the hemoculture-positive individuals; these data are concordant with previous reports, which confirm the predominance of TcII in Brazil ${ }^{3}$. TcI was found in a single six-year-old patient who did not receive blood transfusion and whose mother did not present positive serology for $T$. cruzi. This finding suggests the existence of vector transmission in the studied region. Trypanosoma rangeli was not detected in any of the analyzed samples.

Intense kDNA polymorphism with only $46 \%$ of the bands shared among the TcII populations (mean number of bands, $9.26 \pm 2.68$ ) was demonstrated by LSSP-PCR analysis (Figure 1), which agrees with previous reports ${ }^{3,4,10}$. High genetic variability has previously been detected by LSSP-PCR among TcI stocks ${ }^{11,12}$.

It has been demonstrated that the percentage of polyclonal T. cruzi populations progressively decreases during the chronic phase of Chagas disease ${ }^{1}$. Therefore, for a single endemic area, the $T$. cruzi populations isolated from younger patients should present complex genetic profiles. In the present study, a weak but not significant $(\mathrm{p}>0.05)$ inverse correlation $(\mathrm{r}=-0.3)$ was observed between patient age and the complexity level of the genetic profiles of kDNA obtained by LSSP-PCR. This result may be related to the small number of patients in the youngest age group, from zero to 20 years $(n=5)$.

Despite the high polymorphism of TcII isolates, very similar genetic profiles were observed between specific pairs of T. cruzi samples, particularly for eight pairs of patients with very close degrees of kinship. The isolate pairs $34 / 35,3 / 4$, and $14 / 15$ corresponded to mother-child pairs and shared $92 \%$, $100 \%$, and $100 \%$ of their bands, respectively. The pairs $16 / 22$, $1 / 2$, and $10 / 11$ corresponded to siblings and presented band similarities of $80 \%, 90 \%$, and $96 \%$, respectively. The pairs $8 / 9$ and $16 / 17$ were cousins and shared $92 \%$ and $94 \%$ of their bands, respectively (Figure 1). It is important to emphasize that the electrophoretic kDNA profiles were reproducible for all samples when a second LSSP-PCR was performed. 


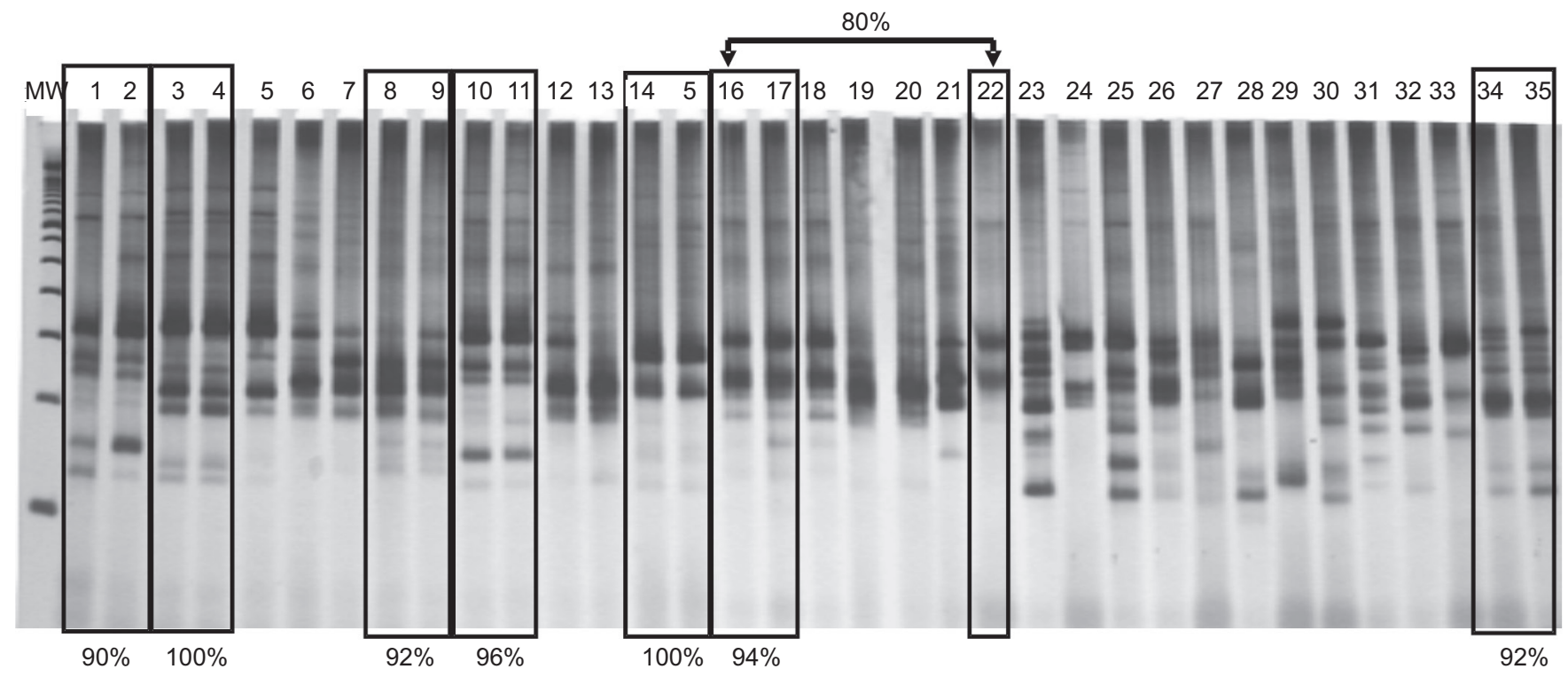

FIGURE 1- Polyacrylamide gel (7.5\%) stained with silver, representing the genetic profiles of Trypanosoma cruzi isolates obtained using LSSP-PCR. The percentages of bands shared between each pair of patients (highlighted) and their level of kinship are as follows: pairs 8/9 (92\%) and 16/17 (94\%) were cousins; pairs 1/2 (90\%), 10/11 (96\%), and 16/22 (94\%) were siblings; pairs 3/4 (100\%), 14/15 $(\mathbf{1 0 0} \%)$, and 34/35 (92\%) were mothers and children. MW: molecular weight (100 bp); LSSP-PCR: low-stringency single-specific-primerpolymerase chain reaction.

Most of the data reported in the literature have demonstrated unique and exclusive LSSP-PCR profiles for individual patients ${ }^{4,10,13,14}$, although no report has described the differential distribution of $T$. cruzi populations in tissue and peripheral blood samples obtained from the same human host ${ }^{1}$. Here, the high similarity of $T$. cruzi kDNA minicircles associated with kinship suggests the possibility of congenital transmission and/or the presence of similar or identical T. cruzi blood populations circulating within the same home or family group. This hypothesis can be supported by evidence of nearly identical patterns of kDNA minicircles between each mother and infant in congenital transmission described in a study using other molecular techniques ${ }^{15}$.

Similarities among the LSSP-PCR T. cruzi genetic profiles were also observed among different individuals infected with TcI from the same geographical region ${ }^{11}$, although it was not possible to associate the kDNA genetic profile with the geographical or biological origin of the studied samples.

These data reinforce the potential of the LSSP-PCR technique for field studies of human Chagas disease and show the importance of using different genetic markers of T. cruzi to monitor the transmission of human Chagas disease, particularly for endemic areas that have been certified as free from vector transmission by Triatoma infestans. These findings also suggest the existence of vector transmission in the study area and may represent an important warning sign for Brazilian epidemiological surveillance programs.

The detection of $T$. cruzi populations that are genetically related and associated with a high degree of kinship, i.e., children and their mothers, is a new approach in the molecular epidemiology of Chagas disease and may provide new strategies for future studies of T. cruzi congenital transmission.

\section{CONFLICT OF INTEREST}

The authors declare that there is no conflict of interest.

\section{FINANCIAL SUPPORT}

This study was supported by grants from the Conselho Nacional de Desenvolvimento Científico e Tecnológico (CNPq): case n ${ }^{\circ}$ 307153/2009-6; Universal Announcement Ministério da Ciência, Tecnologia e Inovação (MCTI): MCT/ CNPq 14/2008 (case n 481231/2008-0); and Coordenação de Aperfeiçoamento de Pessoal de Nivel Superior (CAPES): 14/2013 (case $n^{\circ}$ 481068/2013-9).

\section{REFERENCES}

1. Macedo AM, Machado CR, Oliveira RP, Pena SD. Trypanosoma cruzi: genetic structure of populations and relevance of genetic variability to the pathogenesis of chagas disease. Mem Inst Oswaldo Cruz 2004; 99:1-12.

2. Zingales B, Andrade SG, Briones MR, Campbell DA, Chiari E, Fernandes O, et al. A new consensus for Trypanosoma cruzi intraspecific nomenclature: second revision meeting recommends $\mathrm{TcI}$ to $\mathrm{TcVI}$. Mem Inst Oswaldo Cruz 2009; 104:1051-1054.

3. Zingales B, Miles MA, Campbell DA, Tibayrenc M, Macedo AM, Teixeira MM, et al. The revised Trypanosoma cruzi subspecific 
nomenclature: rationale, epidemiological relevance and research applications. Infect Genet Evol 2012; 12:240-253.

4. Vago AR, Macedo AM, Oliveira RP, Andrade LO, Chiari E, Galvão LMC, et al. Kinetoplast DNA signatures of Trypanosoma cruzi strains obtained directy from infected tissues. Am J Pathol 1996; 149:2153-2159.

5. Chiari E, Dias JCP, Lana M, Chiari CA. Hemocultures for the parasitological diagnosis of human chronic Chagas'disease. Rev Soc Bras Med Trop 1989; 22:19-23.

6. Sambrook J, Fritsch EF, Maniatis T. Molecular Cloning: Laboratory Manual, $2^{\text {th }}$ ed. Book 01. New York: Cold Spring Harbor Laboratory Press; 1989.

7. Gomes ML, Macedo AM, Vago AR, Pena SD, Galvão LMC, Chiari E. Trypanosoma cruzi: optimization of polymerase chain reaction for detection in human blood. Exp Parasitol 1998; 88:28-33.

8. Burgos JM, Altcheh J, Bisio M, Duffy T, Valadares HM, Seidenstein ME, et al. Direct molecular profiling of minicircle signatures and lineages of Trypanosoma cruzi bloodstream populations causing congenital Chagas disease. Int J Parasitol 2007; 37:1319-1327.

9. Souto RP, Vargas N, Zingales B. Trypanosoma rangeli: discrimination from Trypanosoma cruzi based on a variable domain from the large subunit ribosomal RNA gene. Exp Parasitol 1999; 91:306-314.

10. Lages-Silva E, Ramirez LE, Pedrosa AL, Crema E, Galvão LM, Pena SDJ, et al. Variability of kinetoplast DNA gene signatures of
Trypanosoma cruzi II strains from patients with different clinical forms of Chagas' disease in Brazil. J Clin Microbiol 2006; 44:2167-2171.

11. Rodríguez IB, Botero A, Mejía-Jaramillo AM, Marquez EJ, Ortiz S, Solari A, et al. Transmission dynamics of Trypanosoma cruzi determined by low-stringency single primer polymerase chain reaction and southern blot analyses in four indigenous communities of the Sierra Nevada de Santa Marta, Colombia. Am J Trop Med Hyg 2009; 81:396-403.

12. Salazar A, Schijman AG, Triana-Chávez O. High variability of Colombian Trypanosoma cruzi lineage I stocks as revealed by lowstringency single primer-PCR minicircle signatures. Acta Trop 2006; 100:110-118.

13. Mejía-Jaramillo AM, Arboleda-Sánchez S, Rodríguez IB, Cura C, Salazar A, Del Mazo J, et al. Geographical clustering of Trypanosoma cruzi I groups from Colombia revealed by low-stringency single specific primer-PCR of the intergenic regions of spliced-leader genes. Parasitol Res 2009; 104:399-410.

14. Vago AR, Andrade LO, Leite AA, Reis DA, Macedo AM, Adad SJ, et al. Genetic characterization of Trypanosoma cruzi directly from tissues of patients with chronic Chagas disease. Am J Pathol 2000; 156:1805-1809.

15. Burgos JM, Begher SB, Freitas JM, Bisio M, Duffy T, Altcheh J, et al. Molecular diagnosis and typing of Trypanosoma cruzi populations and lineages in cerebral Chagas disease in a patient with AIDS. Am J Trop Med Hyg 2005; 73:1016-1018. 